\title{
An anatomically-based masking protocol for the assessment of in-shoe plantar pressure measurement of the forefoot
}

\author{
Saeed Forghany ${ }^{1,2}$, Daniel R. Bonanno ${ }^{3,4}$, Hylton B. Menz ${ }^{3,4}$ and Karl B. Landorf ${ }^{3,4^{*}}$ (D)
}

\begin{abstract}
Background: The area beneath the metatarsal heads is a common location of foot pain, which is often associated with high plantar pressures. Current plantar pressure assessment protocols focus mainly on the gross area of the forefoot with minimal attention paid to specific areas such as the metatarsal heads. The aim of this study was to develop and assess a new anatomically-based masking protocol that is clinically relevant to measure forefoot plantar pressure during shod conditions based on the anatomical positions of the metatarsal heads.
\end{abstract}

Methods: Initially, we developed a masking protocol to measure forefoot plantar pressure during shod conditions based on the anatomical positions of the metatarsal heads. This new masking protocol divided the forefoot into three sub-areas (proximal, beneath, and distal to the metatarsal heads) as determined by the position of each metatarsal head. Following development of the new masking protocol, we compared the new protocol against a traditional protocol, which defines the forefoot as between 51 and $81 \%$ of the foot length. To compare the two masking protocols, we tested two experimental conditions: (i) a control condition (i.e. no metatarsal pad), and (ii) a metatarsal pad condition. We then compared plantar pressure differences between the two experimental conditions for the two masking protocols. Participants for this component of the study included 36 community dwelling older adults (mean age 75.6 years \pm 5.4 ) with a history of forefoot pain. Forefoot plantar pressure data were measured while walking using the pedar $-X$ in-shoe system. Peak pressure, maximum force and contact area at the time of peak pressure were determined and results were compared between the two masking protocols.

Results: The traditional masking protocol showed that the metatarsal pad significantly decreased peak pressure and increased contact area in the forefoot area (i.e. within the entire mask area), but maximum force was not significantly different between the two conditions. In contrast, the newly developed anatomically-based masking protocol indicated that the metatarsal pad decreased peak plantar pressures distal to and beneath the metatarsal heads by increasing force and contact area proximal to the metatarsal heads.

Conclusions: An anatomically-based masking protocol that is clinically relevant was developed to assess forefoot plantar pressure during shod conditions based on the anatomical positions of metatarsal heads. We propose that the new forefoot masking protocol will provide greater interpretability of forefoot plantar pressure data, which will aid clinicians and researchers for diagnostic, prognostic and therapeutic purposes.

Keywords: Forefoot, Gait, Kinetics, Orthotic devices

\footnotetext{
* Correspondence: k.landorf@latrobe.edu.au

${ }^{3}$ Discipline of Podiatry, School of Allied Health, La Trobe University,

Melbourne, VIC 3086, Australia

${ }^{4}$ La Trobe Sport and Exercise Medicine Research Centre, School of Allied

Health, La Trobe University, Melbourne, VIC 3086, Australia

Full list of author information is available at the end of the article
}

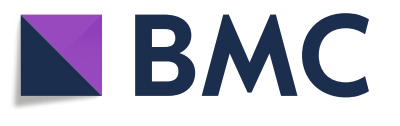

(c) The Author(s). 2018 Open Access This article is distributed under the terms of the Creative Commons Attribution 4.0 International License (http://creativecommons.org/licenses/by/4.0/), which permits unrestricted use, distribution, and reproduction in any medium, provided you give appropriate credit to the original author(s) and the source, provide a link to the Creative Commons license, and indicate if changes were made. The Creative Commons Public Domain Dedication waiver (http://creativecommons.org/publicdomain/zero/1.0/) applies to the data made available in this article, unless otherwise stated. 


\section{Background}

The area beneath the metatarsal heads is a common location of forefoot pain, calluses and neuropathic foot ulcers, and such conditions are often associated with high plantar pressures [1-4]. Accordingly, plantar pressure measurement is frequently recommended as a tool to guide diagnosis, treatment and research investigation for such conditions [5]. There is a relatively large volume of literature that relates to plantar pressure characteristics in the areas of the metatarsal heads, which also includes plantar pressure changes due to treatment of these conditions [2, 6-10]. However, variability in the selection of areas under the metatarsal heads to be investigated on plantar pressure images - referred to as masking - makes explaining plantar pressure changes difficult.

In most plantar pressure studies, the forefoot is selected as a percentage of the plantar pressure insole's length $[7,8,11-15]$. This technique has been claimed to have been determined from skeletal anatomy, but evidence for these percentage measures is sparse. These percentage measures could also be misleading in the presence of foot deformities and during shod conditions, as the pressure measuring insoles are often a different length to the actual foot length being investigated (i.e. they are matched to shoe length rather than foot length). As such, the data from such studies could be ambiguous as it is not clear exactly which part of the foot the pressure results reflect, particularly in relation to the metatarsal heads.

An alternative method of anatomical masking of plantar pressure images involves the synchronisation of a pressure platform with a 3-dimensional motion capture system [16, 17]. Reflective markers are placed on relevant anatomical landmarks on the foot (e.g. the metatarsal heads) and the position of each marker is projected vertically onto the plantar pressure images. Sub-divisions of the foot are then determined based on the position of the markers. However, this method is unlikely to be feasible in clinical scenarios due to its complexity and the high cost of setting up such a system.

Clearly, further research is needed to develop and test appropriate techniques for masking the forefoot to ensure that the most useable information is gained from plantar pressure assessment. These techniques also need to be relatively easy to perform and not be reliant on complex and expensive laboratory techniques. The aim of this study was to develop and assess a new anatomically-based forefoot masking protocol (based on the anatomical positions of the metatarsal heads) that is clinically relevant to measure plantar pressure during shod conditions. To achieve this aim, the effect of a metatarsal pad on forefoot plantar pressure was investigated to evaluate the new masking protocol.

\section{Methods}

The methods for this study are broken into two distinct sections. Initially, the development of the new masking protocol is outlined. Following this, the assessment of the new protocol (reliability and comparison with the traditional masking protocol) is detailed.

\section{Development of the new masking protocol}

For the purpose of developing the new anatomically-based masking protocol, the following method was used. Firstly, each participant's shoe size was determined and they were fitted with a pair of standard extra-depth shoes (Gadean', Perth, Australia). Secondly, following determination of the correct shoe size, a pre-made thin $(<1.0 \mathrm{~mm})$, flexible cardboard insole (also referred to as a template) was fitted to the shoe; multiple templates were pre-made for different shoe sizes. Minor adjustments were made with scissors to ensure that the dimensions of the cardboard insole matched the shoe.

The thin cardboard insole was made for each participant for the purpose of determining the location of the metatarsal heads relative to the insole of the shoe. Accordingly, after fitting the cardboard insole, the metatarsal heads were then palpated and marked with an ink pen on the plantar skin surface, similar to the technique validated by Spooner and colleagues [18]. The participant then placed their feet inside the shoes (i.e. with the cardboard insole inside) and with the laces fastened, the participant was asked to stand to allow the ink to transfer from the foot to the cardboard insole. This process ensured that the position of each metatarsal head (i.e. the inked mark) was transferred to the cardboard insole. The participant was then asked to remove the shoes so that the outline of the metatarsal parabola could be marked on the cardboard insole (from the position of the metatarsal heads outlined by the ink marks).

Once the cardboard insole was marked, the positions of each metatarsal head relative to the insole length was determined. To achieve this, we developed the following standardised protocol.

\section{Step (i)}

To begin, a lateral reference line was determined so each cardboard insole could be aligned in a standardised position (Fig. 1). The lateral reference line was created by drawing a line between two points: (i) a point representing the most lateral aspect of the heel, and (ii) a point representing the most lateral aspect of the forefoot of the insole. This line is represented by line AB in Fig. 1, step (i).

\section{Step (ii)}

Next, a medial to lateral heel line was added to the cardboard insole. With the insole positioned in the standardised position outlined above, the medial to lateral heel line was drawn perpendicular to the lateral reference line at a point $3 \mathrm{cms}$ distal to the most proximal aspect of the heel. This line is represented by line DE in Fig. 1, step (ii). 


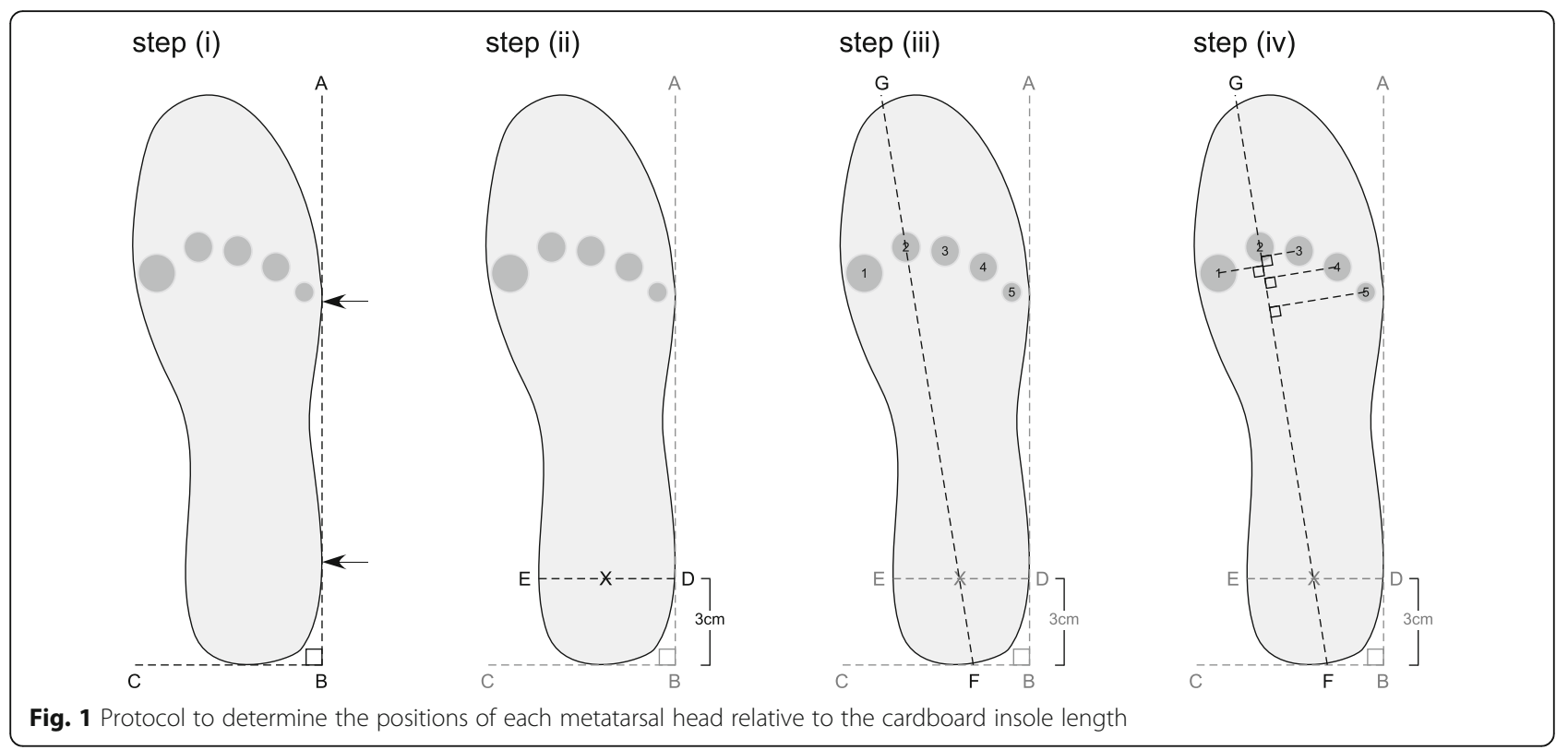

The middle of the heel was then defined as the mid-point along the medial to lateral heel line.

\section{Step (iii)}

Next, a line representing a longitudinal axis line was added to the cardboard insole. This line was established by passing a straight line along the length of the insole that passed through the mid-point of the heel line and orientated distally through the second metatarsal head. This line is represented by line FG in Fig. 1, step (iii). The length of the insole was then measured along the longitudinal axis from the most proximal point (point $F$ ) to the most distal point (Point G) of the template.

\section{Step (iv)}

Finally, the relative position of each metatarsal head was projected onto the longitudinal axis line and their position relative to the cardboard insole length (i.e. relative length) was quantified. To achieve this, the middle of each metatarsal head was marked and a line was drawn from this point so that it intersected with the longitudinal axis at a right angle. This step is represented in Fig. 1, step (iv).

\section{Participants}

Thirty six community-dwelling older people aged 65 or older with the history of forefoot pain were recruited. Participants were recruited and tested between March and November 2013. Ethics approval was obtained from the La Trobe University Faculty Human Ethics Committee - application FHEC12/207. All participants signed informed consent prior to recruitment into the study.

\section{Sample size determination}

The sample size was determined prior to conducting the study (i.e. a priori) using an appropriate formula [19]. A sample size of 36 provides an $80 \%$ probability of detecting a clinically meaningful difference between interventions of 60 kilopascals $(\mathrm{kPa})$ in peak plantar pressure. The standard deviation used to determine this sample size was taken from a similar studies that measured plantar pressures in older people $[7,8,20,21]$ and was set at $90 \mathrm{kPa}$. The alpha level was set at 0.05 .

\section{Assessment of the new masking protocol}

To assess the new masking protocol, a commercially available prefabricated metatarsal pad constructed from $\mathrm{PPT}^{\circ}$ - a proprietary medical grade soft-tissue supplement - was used (Langer Biomechanics, New York, USA). This pad is teardrop shaped and is $6 \mathrm{~mm}$ at the highest point. It is a commonly used pad to alleviate plantar forefoot pain and the supplier of the pads for this study indicated that it is the most commonly ordered metatarsal pad from their company by podiatrists in Australia (personal communication, Mark Dannals, Briggate Medical Company). The pad was dispensed in one of two different sizes (small or medium) depending on foot size. When the metatarsal pad was tested, it was adhered to the cardboard insole using double-sided adhesive tape to prevent the pad from moving during testing. The cardboard insole was positioned between the plantar surface of the foot and the inside of the sole of the shoe. There were two conditions tested in this study:

(i) control (no pad) condition; 
(ii) metatarsal pad positioned in line with the metatarsal heads (Fig. 2).

The order of testing was randomised to avoid ordering effects. In addition, we attempted to blind participants as to the types of interventions used (this was part of a larger study where multiple pads were evaluated), so participants were not informed of the study purpose, other than that we were evaluating how forefoot pads change pressure under the foot. Accordingly, participants were not advised of the study hypotheses.

Plantar pressures beneath the foot were measured using the pedar-X in-shoe plantar pressure system (Novel $\mathrm{GmbH}$, Munich, Germany). The pedar-X comprises of 99 capacitive sensors arranged in a grid and embedded within a thin flexible insole. The pedar $-\mathrm{X}$ insoles were calibrated using the trublu calibration device (Novel GmbH, Munich, Germany) [22] prior to data collection. The sampling frequency of the system was $50 \mathrm{~Hz}$. The pedar- $-\mathrm{X}$ is widely used in foot plantar pressure research [6, 21, 23-25] and has been demonstrated to be a valid and reliable in-shoe pressure measurement system [26-29]. It has high test-retest reliability with coefficients of repeatability for metatarsal head measurements of between 1.2 to $7.7 \%$ [28], and coefficients of variation for metatarsal head measurements of between 3.4 and $24.1 \%$ [29]. This equipment

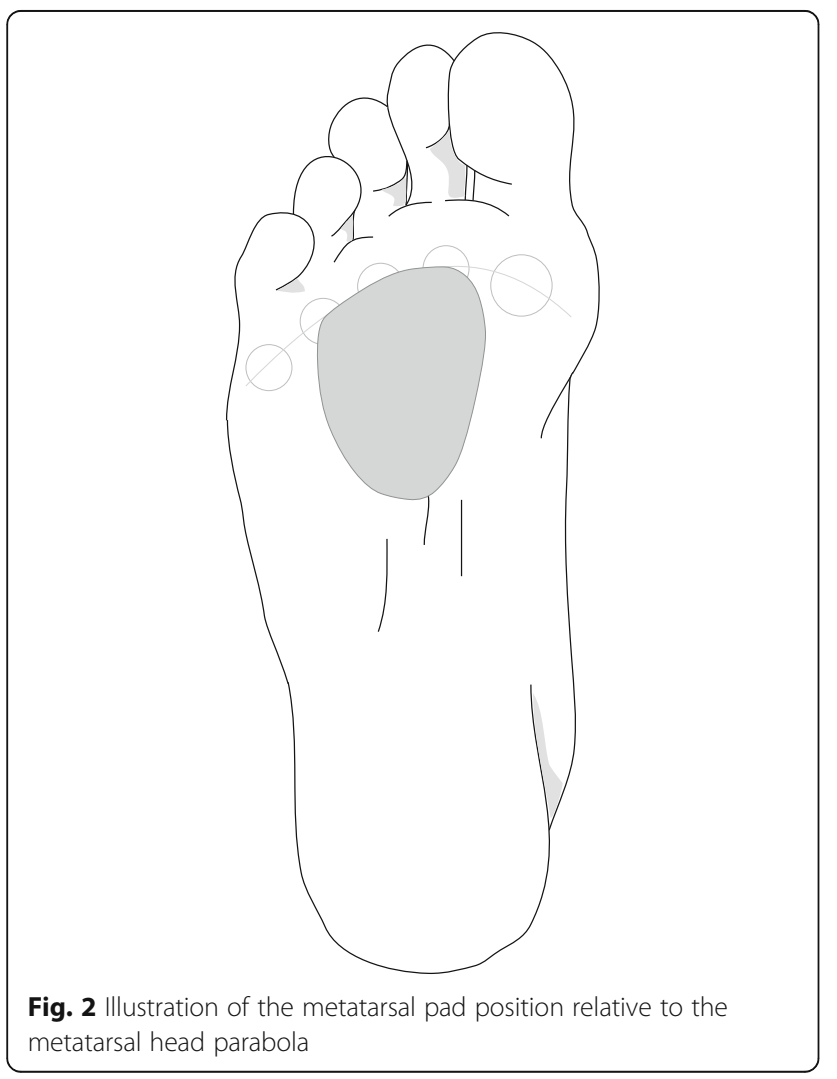

has also previously been used in similar projects with older people [7, 8, 20, 21].

The appropriately sized pedar $-\mathrm{X}$ insole was inserted into the shoe on top of the cardboard insole, and then the participant put the shoes on again, so the pedar $-\mathrm{X}$ insole was positioned between the participant's foot and the cardboard insole with the metatarsal pad. The participant was then instructed to walk at their normal comfortable speed while being timed. To minimise the confounding effect of different walking speeds on the pressure data, the trial was repeated if the walking speed differed by more than $5 \%$ of the original walking speed. Four walking trials along an $8 \mathrm{~m}$ walkway were recorded for each test condition, with the middle 4 steps for each trial included in the analysis (to exclude acceleration and deceleration steps). The 16 steps ( 4 trials $\times 4$ steps) were then averaged for each condition.

The forefoot plantar pressure data were then assessed with a traditional masking protocol that uses the percentage of foot length (between 51 and $81 \%$ of foot length) [22]. This was then compared with our new masking protocol (Fig. 3). To determine the new masking protocol, the following process was followed. Once the relative position of each metatarsal head was calculated from the cardboard insole (i.e. \% relative to the insole length), this information was used to determine which sensors on the pedar $-\mathrm{X}$ insole were to be analysed for the 'beneath the metatarsal heads' analysis. Once this was determined, the sensors one row distal to this were chosen for the 'distal to the metatarsal heads' analysis and the sensors proximal to this were chosen for the 'proximal to the metatarsal heads' analysis. Two rows of sensors were chosen for the 'proximal to the metatarsal heads' analysis to allow greater sensor area to detect changes in pressure variables as the metatarsal pad being tested was moved proximally where the arch of the foot was.

The primary outcome measure of interest was peak pressure under the forefoot. The secondary outcomes included maximum force and contact area at the time of peak pressure, which we used to help explain the peak plantar pressure findings. Outcomes were determined using both masking protocols and then the results from each protocol were compared.

\section{Data analysis}

To analyse reliability of the measurements from the cardboard insoles the following was done. Intra-rater reliability was analysed by examining repeated measurements of cardboard insole length and metatarsal head length relative to cardboard insole length. Measurements were taken over two different days on the same cardboard insoles by the same two raters (DRB and KBL). Inter-rater reliability was analysed by measurement of 

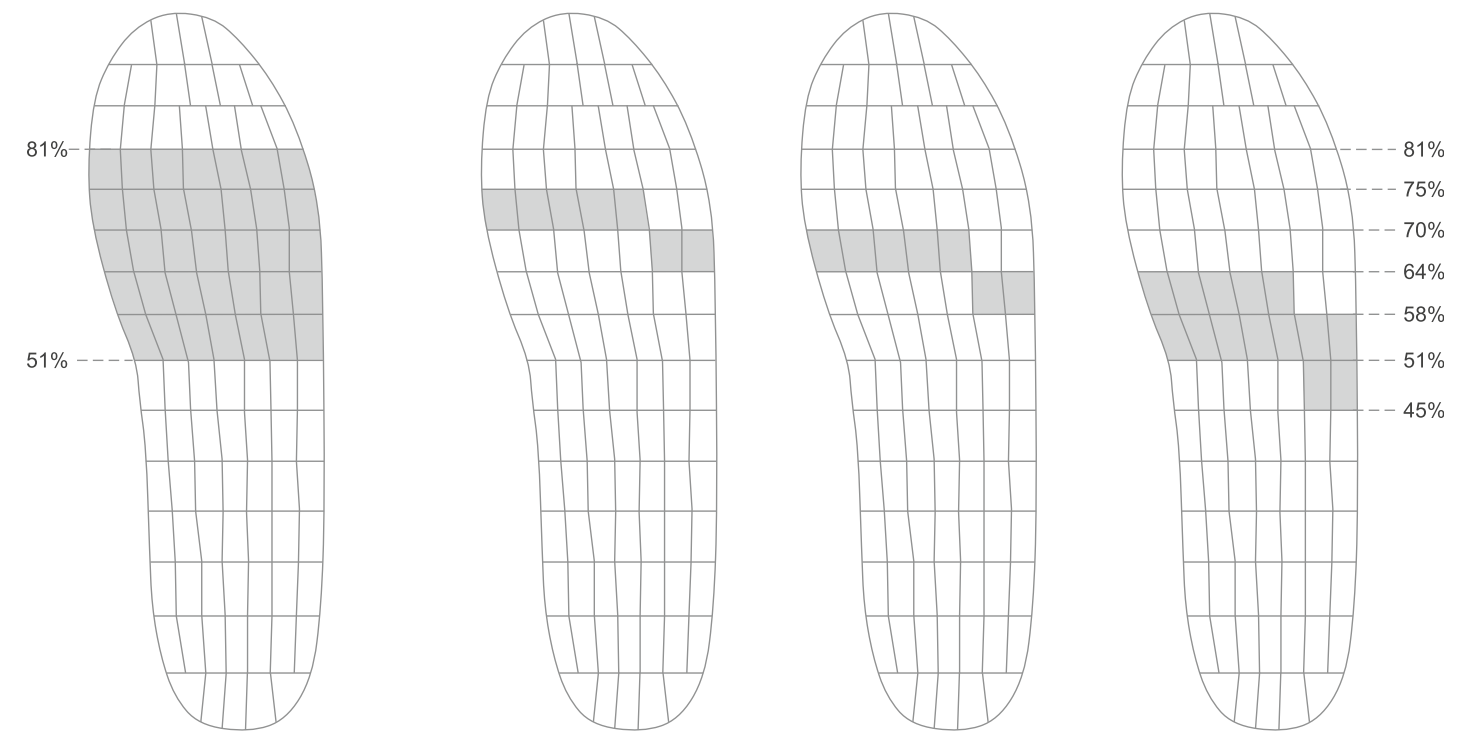

Fig. 3 The traditional masking protocol and the anatomically-based masking protocol (Note: for the anatomically-based protocol, three mask sub-areas of forefoot were selected according to the positions of metatarsal heads: (i) distal to the metatarsal heads, (ii) beneath the metatarsal heads, and (iii) proximal to the metatarsal heads)

cardboard insole length and positions of the metatarsal heads by three different raters (SF, DRB and KBL) who took the measurements independent of each other. Intra-class correlation coefficients (ICCs) were used to determine the reliability coefficients using an ICC $(3,1)$ model [30].

To analyse the plantar pressure data, we firstly checked that the plantar pressure data were normally distributed by assessing skewness, kurtosis, and the Shapiro-Wilk test $(p>0.05)$. To compare the two masking protocols, paired $t$-tests were used to evaluate for differences $(p<0.05)$ between the two conditions (i.e. no pad control condition versus metatarsal pad condition).

IBM SPSS Statistics version 16.0 (IBM Corporation, Armonk, NY, USA) was used for all analyses, except for percentage change and effect size calculations, which were carried out with Microsoft Excel (Microsoft Office 2013) using the formula proposed by Thalheimer and Cook [31]. Effect size magnitudes were categorised according to Hopkins [32].

\section{Results}

\section{Participant characteristics}

The mean age of participants was $75.6 \pm 5.4$ years (range 65.1 to 88.6 years). There were 31 females and 5 males. The mean BMI for participants was $28.3 \pm 4.1 \mathrm{~kg} / \mathrm{m}^{2}$.

\section{Anatomically-based masking protocol}

An anatomically-based masking protocol was developed by dividing the forefoot into three sub-areas as determined by the position of the metatarsal heads. Metatarsal heads were located between $59.7 \pm 1.8 \%$ and $69.9 \pm 1.8 \%$ of cardboard insole length. Reliability analysis indicated that the protocol had excellent intra- and inter-rater reliability. Table 1 presents the ICC values for the intra- and inter-rater reliability analysis for cardboard insole length and metatarsal heads positions.

Table 2 presents the mean position of each metatarsal head as a percentage of the overall cardboard insole length.

Three mask sub-areas of forefoot were selected according to the positions of metatarsal heads: (i) distal to the metatarsal heads, (ii) beneath the metatarsal heads, and (iii) proximal to the metatarsal heads. The traditional masking protocol and the anatomically-based masking protocol, including the three mask sub-areas, are illustrated in Fig. 3.

\section{Traditional masking protocol versus anatomically-based masking protocol}

In relation to peak pressure (Table 3), analysing data using the traditional masking protocol showed that the metatarsal pad (compared to the control condition) significantly reduced peak pressure in the forefoot $(-14.3 \%, p<0.001$, effect size medium 0.55). Analysing data using the anatomically-based masking protocol showed that the 
Table 1 ICC values of between-day and between-tester for cardboard insole length and metatarsal head positions

\begin{tabular}{llllll}
\hline & R1D1 vs R1D2 & R2D1 vs R2D2 & R1D1 vs R2D1 & R1D2 vs R2D2 & R1D1 vs R2D1 vs R3D1 \\
\hline Insole length & 0.989 & 0.998 & 0.987 & 0993 & 0.995 \\
Metatarsal head 1 & 0.964 & 0.996 & 0.966 & 0.987 & 0.982 \\
Metatarsal head 2 & 0.990 & 0.998 & 0.985 & 0.991 & 0.989 \\
Metatarsal head 3 & 0.988 & 0.996 & 0.988 & 0.988 & 0.993 \\
Metatarsal head 4 & 0.981 & 0.988 & 0.983 & 0.973 & 0.980 \\
Metatarsal head 5 & 0.983 & 0.991 & 0.984 & 0.979 & 0.967
\end{tabular}

$R 1$ Rater 1, R2 Rater 2, R3 Rater 3,D1 Day 1, D2 Day 2

metatarsal pad (compared to the control condition) significantly reduced peak pressure in the forefoot mask sub-areas distal to the metatarsal heads $(-17.0 \%$ change, $p<0.001$, effect size medium 0.55) and beneath the metatarsal heads $3(-17.3 \%$ change, $p=0.004$, effect size medium 0.53). However, peak pressure did not change significantly in the forefoot mask sub-area proximal to the metatarsal heads $(p=0.333)$.

In relation to maximum force at the time of peak pressure (Table 4), analysing data using the traditional masking protocol showed that the metatarsal pad (compared to the control condition) did not change maximum force significantly in the forefoot $(p=0.115)$. Analysing data using the anatomically-based protocol showed that the metatarsal pad (compared to the control condition) significantly reduced maximum force at the time of peak pressure in the forefoot mask sub-areas distal to the metatarsal heads $(-9.9 \%$ change, $p<0.001$, effect size medium $0.51)$ and beneath the metatarsal heads ( $-16.6 \%$ change, $p=0.001$, effect size medium 0.55 ). There was also a significant increase of maximum force at the time of peak pressure in the forefoot mask sub-area proximal to the metatarsal heads $(+24.5 \%$ change, $p=0.048$, effect size small 0.38).

In relation to contact area at the time of peak pressure (Table 5), analysing data using the traditional masking protocol showed that the metatarsal pad (compared to the control condition) significantly increased contact area in the forefoot $(+8.1 \%$ change, $p<0.001$, effect size medium $0.61)$. Analysing data using the anatomically-based protocol showed that the metatarsal pad (compared to the control condition) did not change the contact area significantly in the forefoot mask sub-area distal to the metatarsal heads $(p=0.142)$, but significantly reduced contact area in the forefoot mask sub-area beneath the metatarsal heads $(-2.3 \%, p=0.033$, effect size small 0.31$)$ and significantly increased contact area in the forefoot mask sub-area proximal to the metatarsal heads $(+21.8 \%$, $p<0.001$, effect size medium 0.62).

\section{Discussion}

To address the methodological shortcomings of existing plantar pressure masking protocols, a new masking protocol was developed to be able to better interpret forefoot plantar pressure during shod conditions based on the anatomical positions of the metatarsal heads. This method allows plantar pressure parameters to be determined proximal, beneath and distal to the metatarsal heads. Our new protocol is tailored to the anatomy of a person's foot rather than the size of the plantar pressure insole used to assess that person. Importantly, we found that our new masking protocol has excellent intra- and inter-rater reliability.

To highlight the clinical relevance of this method and to help validate our protocol, we focused on the example of forefoot pain in our study. Forefoot pads are often used as they have been shown to reduce plantar pressures under the metatarsal heads, which can alleviate pain under the forefoot. However, the precise mechanism as to how forefoot pads achieve this has remained largely unexplained in the current literature. Research thus far has not been able to accurately map the changes in force and contact area that lead to these plantar pressure changes. Doing so would help understand how forefoot pads work, which may lead to the development of more effective pads.

Our findings also highlight critical deficiencies in the results of plantar pressure measures obtained from traditional masking protocols. When analysing plantar pressure data using the traditional masking protocol, we were not able to determine the effects of the metatarsal pad from the perspective of force and contact area changes. However, analysing pressure data using our new anatomically-based masking showed how a metatarsal pad redistributes

Table 2 Position of each metatarsal head as the percentage of cardboard insole length (values are means \pm SDs)

\begin{tabular}{llllll}
\hline & Metatarsal head 1 & Metatarsal head 2 & Metatarsal head 3 & Metatarsal head 4 & Metatarsal head 5 \\
\hline Position (\% of cardboard insole length) & $69.3 \pm 1.9$ & $69.9 \pm 1.8$ & $67.7 \pm 1.7$ & $64.0 \pm 1.6$ & $59.7 \pm 1.8$ \\
\hline
\end{tabular}


Table 3 Peak pressure ( $\mathrm{kPa}$ ) using two different masking protocols and during two insole conditions (values are means \pm SDs, percentage changes and effect sizes)

\begin{tabular}{|c|c|c|c|c|c|}
\hline & & \multicolumn{2}{|c|}{ Insole condition } & \multirow[t]{2}{*}{ \% change (95\% Cl) } & \multirow[t]{2}{*}{ Effect size } \\
\hline & & Control & Pad in-line & & \\
\hline Traditional mask & & $389.4 \pm 113.6$ & $333.6 \pm 91.2$ & $-14.3 \%(-20.0$ to $-8.7 \%)$ & $0.55^{*}$ \\
\hline \multirow[t]{3}{*}{ Anatomically-based mask } & Distal & $351.7 \pm 127.5$ & $292.0 \pm 99.0$ & $-17.0 \%(-24.0$ to $-9.9 \%)$ & $0.53^{*}$ \\
\hline & Beneath & $213.8 \pm 90.3$ & $176.8 \pm 64.3$ & $-17.3 \%(-28.5$ to $-6.1 \%)$ & $0.48^{*}$ \\
\hline & Proximal & $110.9 \pm 52.4$ & $104.7 \pm 27.7$ & $-5.6 \%(-17.3$ to $6.0 \%)$ & 0.15 \\
\hline
\end{tabular}

Note: Control insole condition indicates no metatarsal pad and pad-in-line condition indicates metatarsal pad positioned in-line with the metatarsal head parabola. *Significantly different peak pressure for the pad in-line condition compared with the control condition, $p<0.05$

plantar pressure - via force and contact area changes - in the forefoot area, particularly in relation to the metatarsal heads. Specifically, the metatarsal pad decreases peak plantar pressures distal to and beneath the metatarsal heads by increasing force and contact area proximal to the metatarsal heads. This highlights the importance of appropriate masking to explain plantar pressure changes in the forefoot and has the potential for more objective evaluation of forefoot problems and interventions for these problems. Our anatomically-based masking will enable researchers and clinicians to target the appropriate area of the plantar surface of the forefoot during research, rehabilitation, and the prescription, design and evaluation of footwear and foot orthoses.

Furthermore, our individualised plantar pressure masking protocol is in-line with individualised orthotic management [33]. Ideally, a forefoot pad would be positioned and assessed using plantar pressure data that is specific to an individual. Doing so should more effectively relieve pain and protect vulnerable tissues. Indeed, the Guidance on footwear and offloading 2015 document published by the International Working Group on the Diabetic Foot recommends such an approach [34]. Although we did not evaluate patient-reported outcomes, it is plausible that taking such an individualised approach may also improve function, which could lead to benefits to overall foot health status. Such a hypothesis needs to be evaluated in randomised trials.

Our new anatomically-based masking protocol provides a robust technique to measure the positions of the metatarsal heads. Even though we only measured the positions of the metatarsal heads in this study, the proposed method could be extended to the determination of other anatomical landmarks of the plantar surface of the foot (e.g. the styloid process or the medial calcaneal tubercle).

One of most important advantages of plantar pressure measurement devices compared to other gait analysis tools is that they are pre-calibrated, easy to set-up, and portable. Some studies have proposed synchronisation of a pressure platform with a 3-dimensional motion capture system for the purpose of masking $[16,17]$. However, this method is cumbersome, especially in clinical scenarios, due to the high costs of set-up and complexity. Further, when using this method the sub-divisions of the foot are determined based on the position of skin markers, but this introduces limitations in marker set-up.

There are four limitations to our new forefoot masking protocol. Firstly, the accuracy of our masking protocol depends on the spatial resolution of the plantar pressure insoles, particularly given that we are using relatively small masking areas [35]. Insoles with large sensors may not offer sufficient spatial resolution, which may render the process inaccurate. This is an inherent limitation of currently available plantar pressure measuring insole technology and can only be solved by manufacturers of such systems improving the spatial resolution by increasing the number of sensors per insole. Indeed, platform-based plantar pressure systems, such as the emed, do just that they have better spatial resolution because they have more

Table 4 Maximum force at the time of peak pressure (Newtons) using two different masking protocols and during two insole conditions (values are means \pm SDs, percentage changes and effect sizes)

\begin{tabular}{|c|c|c|c|c|c|}
\hline & & \multicolumn{2}{|c|}{ Insole condition } & \multirow[t]{2}{*}{ \% change (95\% Cl) } & \multirow[t]{2}{*}{ Effect size } \\
\hline & & Control & Pad in-line & & \\
\hline Traditional mask & & $518.6 \pm 102.1$ & $504.4 \pm 91.9$ & $-2.7 \%(-6.2$ to 0.7$)$ & 0.15 \\
\hline \multirow[t]{3}{*}{ Anatomically-based mask } & Distal & $207.3 \pm 43.8$ & $186.7 \pm 37.9$ & $-9.9 \%(-13.7$ to -6.1$)$ & $0.51^{*}$ \\
\hline & Beneath & $134.4 \pm 50.2$ & $112.0 \pm 30.3$ & $-16.6 \%(-26.0$ to -7.3$)$ & $0.55^{*}$ \\
\hline & Proximal & $82.0 \pm 61.1$ & $102.2 \pm 46.2$ & $24.5 \%(0.2$ to 48.9$)$ & $0.38^{*}$ \\
\hline
\end{tabular}

Note: Control insole condition indicates no metatarsal pad and pad-in-line condition indicates metatarsal pad positioned in-line with the metatarsal head parabola. *Significantly different peak pressure for the pad in-line condition compared with the control condition, $p<0.05$ 
Table 5 Contact area $\left(\mathrm{cm}^{2}\right)$ at the time of peak pressure using two different masking protocols and during two insole conditions (values are means \pm SDs, percentage changes and effect sizes)

\begin{tabular}{|c|c|c|c|c|c|}
\hline & & \multicolumn{2}{|c|}{ Insole condition } & \multirow[t]{2}{*}{$\%$ change $(95 \% \mathrm{Cl})$} & \multirow[t]{2}{*}{ Effect size } \\
\hline & & Control & Pad in-line & & \\
\hline Traditional mask & & $40.3 \pm 5.9$ & $43.6 \pm 5.0$ & $8.1 \%(4.7$ to 11.5$)$ & $0.61^{*}$ \\
\hline \multirow[t]{3}{*}{ Anatomically-based mask } & Distal & $10.7 \pm 0.9$ & $10.8 \pm 0.7$ & $1.2 \%(-0.4$ to 2.9$)$ & 0.13 \\
\hline & Beneath & $10.1 \pm 0.6$ & $9.9 \pm 0.7$ & $-2.3 \%(-4.4$ to -0.2$)$ & $0.31^{*}$ \\
\hline & Proximal & $14.7 \pm 5.7$ & $17.9 \pm 4.7$ & $21.8 \%$ (10.6 to 33.0$)$ & $0.62^{*}$ \\
\hline
\end{tabular}

Note: Control insole condition indicates no metatarsal pad and pad-in-line condition indicates metatarsal pad positioned in-line with the metatarsal head parabola. *Significantly different peak pressure for the pad in-line condition compared with the control condition, $p<0.05$

sensors per unit area - but they cannot be used for in-shoe insole evaluations. Secondly, the proposed masking protocol depends on the anatomical knowledge of the clinician (i.e. the initial palpation and marking of the metatarsal heads) and the accuracy with which each anatomical landmark is identified. Further accuracy studies investigating multiple clinicians are suggested to validate our new forefoot masking method using a gold-standard such as weight bearing x-rays. Thirdly, we used standardised extra depth footwear for testing participants to control for variation in footwear and to ensure the process of making and fitting the thin cardboard insoles was as efficient as possible. However, this may have an effect on the generalisability of our findings as outside of our study environment, patients would use their own shoes. Finally, our study included a relatively small sample of participants. Studies on larger samples of people with normal foot structure/alignment and those with orthopaedic deformity are suggested to provide more extensive validation of our new protocol. This will enable us to target specific areas of the plantar surface of the foot appropriately.

\section{Conclusion}

An easy to use and anatomically-based masking protocol that is clinically relevant was developed to assess forefoot plantar pressure during shod conditions based on the anatomical positions of metatarsal heads. The new protocol was found to have excellent intra- and inter-rater reliability. We propose that the new forefoot masking protocol will provide greater interpretability of forefoot plantar pressure data, which will aid clinicians and researchers for diagnostic, prognostic, therapeutic and research purposes.

\section{Abbreviations}

kPa: kilopascal

\section{Acknowledgements}

HBM is currently a National Health and Medical Research Council of Australia Senior Research Fellow (ID: 1135995).

\section{Funding}

No funding was obtained for this study, however Briggate Medical Company, Melbourne Australia kindly provided the pads for the study at no cost.
Availability of data and materials

The datasets used and/or analysed during the current study are available from the corresponding author on reasonable request.

\section{Authors' contributions}

SF was involved in study conception and design, data analysis and interpretation, and drafting the manuscript. DRB was involved in study design, data interpretation, and contributed to drafting the manuscript. HBM was involved in study design, data interpretation, and contributed to drafting the manuscript. KBL was involved in study design, securing materials from Briggate Medical Company, data analysis and interpretation, and drafting the manuscript. All authors have read and approved the final manuscript.

\section{Ethics approval and consent to participate}

Ethical approval for the experimental component of this study was provided by the La Trobe University Faculty Human Ethics Committee (Application FHEC12/207). All participants signed informed consent.

\section{Competing interests}

HBM is Editor-in-Chief, DRB is Associate Editor, and KBL is a member of the Editorial Board of Journal of Foot and Ankle Research. It is journal policy that editors are removed from the peer review and editorial decision making processes for papers they have co-authored.

\section{Publisher's Note}

Springer Nature remains neutral with regard to jurisdictional claims in published maps and institutional affiliations.

\section{Author details}

${ }^{1}$ Musculoskeletal Research Centre, School of Rehabilitation Sciences, Isfahan University of Medical Sciences, Isfahan, Iran. ${ }^{2}$ Centre for Health Sciences Research, School Health Sciences, University of Salford, Salford, UK. ${ }^{3}$ Discipline of Podiatry, School of Allied Health, La Trobe University, Melbourne, VIC 3086, Australia. ${ }^{4}$ La Trobe Sport and Exercise Medicine Research Centre, School of Allied Health, La Trobe University, Melbourne, VIC 3086, Australia.

Received: 19 February 2018 Accepted: 25 May 2018

Published online: 15 June 2018

\section{References}

1. Benvenuti F, Ferrucci L, Guralnick JM, Gangemi S, Baroni A. Foot pain and disability in older persons: an epidemiologic survey. J Am Geriatr Soc. 1995; 43(5):479-84.

2. Menz HB, Zammit GV, Munteanu SE. Plantar pressures are higher under callused regions of the foot in older people. Clin Exp Dermatol. 2007;32(4):375-80.

3. Veves A, Murray HJ, Young MJ, Boulton AJ. The risk of foot ulceration in diabetic patients with high foot pressure: a prospective study. Diabetologia. 1992;35(7):660-3.

4. Bus SA, Maas M, de Lange A, Michels RP, Levi M. Elevated plantar pressures in neuropathic diabetic patients with claw/hammer toe deformity. J Biomech. 2005;38(9):1918-25.

5. Williams DA, Nester C. Footwear and foot orthoses. Edinburgh: Churchill Livingstone/Elsevier; 2010. 
6. Burns J, Crosbie J, Ouvrier R, Hunt A. Effective orthotic therapy for the painful Cavus foot: a randomized controlled trial. J Am Podiatr Med Assoc. 2006;96(3):205-11.

7. Lee PY, Landorf KB, Bonanno DR, Menz HB. Comparison of the pressurerelieving properties of various types of forefoot pads in older people with forefoot pain. J Foot Ankle Res. 2014;7:1

8. Lane TJ, Landorf KB, Bonanno DR, Raspovic A, Menz HB. Effects of shoe sole hardness on plantar pressure and comfort in older people with forefoot pain. Gait Posture. 2014;39(1):247-51.

9. Landorf KB, Morrow A, Spink MJ, Nash CL, Novak A, Potter J, Menz HB. Effectiveness of scalpel debridement for painful plantar calluses in older people: a randomized trial. Trials. 2013;14:243.

10. Keijsers N, Stolwijk N, Louwerens J, Duysens J. Classification of forefoot pain based on plantar pressure measurements. Clin Biomech (Bristol, Avon). 2013;28(3):350-6.

11. Zhang X, Li B. Influence of in-shoe heel lifts on plantar pressure and center of pressure in the medial-lateral direction during walking. Gait Posture. 2014;39(4):1012-6.

12. Chang B-C, Liu D-H, Chang JL, Lee S-H, Wang J-Y. Plantar pressure analysis of accommodative insole in older people with metatarsalgia. Gait Posture. 2014;39(1):449-54

13. Forghany S, Nester CJ, Richards B, Hatton AL, Liu A. Rollover footwear affects lower limb biomechanics during walking. Gait Posture. 2014;39(1):205-12.

14. Burns J. The assessment and management of a patient with painful idiopathic pes cavus. Australas Journal Podiatr Med. 2004;38(2):49-53.

15. Smoliga JM, Wirfel LA, Paul D, Doarnberger M, Ford KR. Effects of unweighting and speed on in-shoe regional loading during running on a lower body positive pressure treadmill. J Biomech. 2015;48(10):1950-6.

16. Giacomozzi C, Macellari V, Leardini A, Benedetti M. Integrated pressureforce-kinematics measuring system for the characterisation of plantar foot loading during locomotion. Med Biol Eng Comput. 2000;38(2):156-63.

17. Stebbins JA, Harrington ME, Giacomozzi C, Thompson N, Zavatsky A, Theologis TN. Assessment of sub-division of plantar pressure measurement in children. Gait Posture. 2005;22(4):372-6.

18. Spooner SK, Kilmartin TE, Merriman LM. The palpation technique for determination of metatarsal formula: a study of validity. Foot (Edinb). 1994; 4(4):198-200.

19. Friedman LM, Furberg CD, DeMets DL. Fundamentals of clinical trials. New York: Springer; 2010.

20. Cronkwright DG, Spink MJ, Landorf KB, Menz HB. Evaluation of the pressureredistributing properties of prefabricated foot orthoses in older people after at least 12 months of wear. Gait Posture. 2011;34(4):553-7.

21. Bonanno DR, Landorf KB, Menz HB. Pressure-relieving properties of various shoe inserts in older people with plantar heel pain. Gait Posture. 2011;33(3):385-9.

22. Novel. Novel Scientific Software Manual version 2.0. Munich: Novel GMbH; 2010

23. Guldemond N, Leffers $P$, Schaper N, Sanders A, Nieman F, Willems $P$, Walenkamp $\mathrm{G}$. The effects of insole configurations on forefoot plantar pressure and walking convenience in diabetic patients with neuropathic feet. Clin Biomech (Bristol, Avon). 2007;22(1):81-7.

24. Hennessy K, Burns J, Penkala S. Reducing plantar pressure in rheumatoid arthritis: a comparison of running versus off-the-shelf orthopaedic footwear. Clin Biomech (Bristol, Avon). 2007;22(8):917-23.

25. Redmond AC, Landorf KB, Keenan A-M. Contoured, prefabricated foot orthoses demonstrate comparable mechanical properties to contoured, customised foot orthoses: a plantar pressure study. J Foot Ankle Res. 2009;2:20.

26. Hsiao H, Guan J, Weatherly M. Accuracy and precision of two in-shoe pressure measurement systems. Ergonomics. 2002;45(8):537-55.

27. Murphy DF, Beynnon BD, Michelson JD, Vacek PM. Efficacy of plantar loading parameters during gait in terms of reliability, variability, effect of gender and relationship between contact area and plantar pressure. Foot Ankle Int. 2005;26(2):171-9.

28. Putti A, Arnold G, Cochrane L, Abboud R. The Pedar in-shoe system: repeatability and normal pressure values. Gait Posture. 2007;25(3):401-5.

29. Ramanathan A, Kiran P, Arnold G, Wang W, Abboud R. Repeatability of the Pedar- $X^{\circ}$ in-shoe pressure measuring system. Foot Ankle Surg. 2010;16(2):70-3.

30. Portney LG, Watkins MP. Foundations of clinical research: applications to practice, 3rd edn. Upper Saddle River, NJ: Prentice Hall; 2008.

31. Thalheimer W, Cook S. How to calculate effect sizes from published research articles: a simplified Methodology 2002. [http://www. bwgriffin.com/gsu/courses/edur9131/content/Effect_Sizes_pdf5.pdf] Accessed 2 Feb 2018.
32. Hopkins WG. A scale of magnitudes for effect Statistics 2006. [http://sportsci. org/resource/stats/effectmag.html] Accessed 2 Feb 2018

33. McKee PR, Rivard A. Biopsychosocial approach to orthotic intervention. J Hand Ther. 2011:24(2):155-63.

34. International Working Group on the Diabetic Foot. Guidance on footwear and offloading. 2015. [http://iwgdf.org/] Accessed 2 Feb 2018.

35. Urry S. Plantar pressure-measurement sensors. Meas Sci Technol. 1999; 10(1):R16-32

\section{Ready to submit your research? Choose BMC and benefit from:}

- fast, convenient online submission

- thorough peer review by experienced researchers in your field

- rapid publication on acceptance

- support for research data, including large and complex data types

- gold Open Access which fosters wider collaboration and increased citations

- maximum visibility for your research: over $100 \mathrm{M}$ website views per year

At BMC, research is always in progress.

Learn more biomedcentral.com/submissions 